\title{
Research on High Resolution Stress Corrosion Crack Detection Based on Remote Field Eddy Current Non-Destructive Testing
}

\author{
Xu Xiaojie ${ }^{*}, 1$, Jia Yueling ${ }^{2}$ and Zhang Zhanbin ${ }^{1}$ \\ ${ }^{1}$ Institute of Information and Navigation, Air Force Engineering University Xi'an, 710077, P.R. China \\ ${ }^{2}$ Institute of Equipment Management and Engineering, Air Force Engineering University Xi'an, 710077, P.R. China
}

\begin{abstract}
Although remote field eddy current had achieved successive application in pipes and tubes non-destructive testing, the poor ability and sensitivity to axial crack such as stress corrosion crack prevented its further application. A novel high resolution remote field eddy current detection method which can be suitable for axial crack especially stress corrosion crack detection was proposed and verified by simulation and experiment. First of all, conventional detection ability of remote field eddy current for crack in different directions was researched with detail. Aside from this, applicability of orthogonal magnetic filed in remote field eddy current was analyzed and simulated by finite element method, and appropriate configuration using to generating orthogonal magnetic fields for tubular structure was discussed and verified. Thirdly, design of detection system including key parameters selection and detection coil mode were researched. At last, proposed high resolution detection system were verified experimentally using various type defects, such as parallel axial crack and real stress corrosion crack. From the above research, conclusions were drawn as followed: the detection ability and sensitivity of proposed remote field eddy current detection system could be improved significantly compared with conventional system, especially for axial crack which was not easily to detect, and real stress corrosion crack could be detected and evaluated successively.
\end{abstract}

Keywords: Detection, finite element simulation, non-destructive testing, remote field eddy current, stress corrosion crack.

\section{INTRODUCTION}

Stress corrosion crack (SCC) is typical defect of industry tubular structures such as oil and gas pipes [1]. The main feature of SCC can be summarized as followed: tiny, cluster and often axial oriented. Non-Destructive Testing (NDT) is an effective method to detect it and avoid the occurrence of accident. Remote field eddy current (RFEC) is a "typical eddy current NDT method, which has advantages such as almost equal sensitivity to inner and outer defects, easy defect characterization and insensitivity to lift-off or wobble" $[2,3]$. Principle of RFEC can be seen form Fig. (1). Remote field effect begins from where wave outside the pipe overruns it inside the pipe [4].

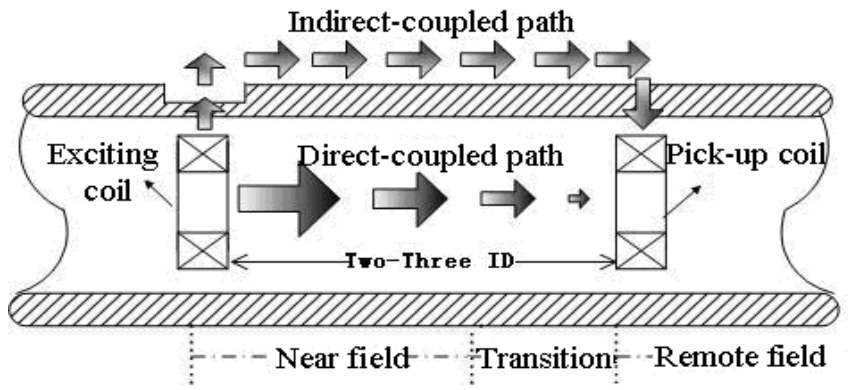

Fig. (1). Illustration of RFEC.
Although RFEC can detect many type defects of pipes and tubes, poor ability to detect SCC prevents its further application. It is mainly because of that conventional RFEC sensor using two solenoid coils as exciting and detection electromagnetic disturbance, and is more sensitive to circumferential defect than axial defect. Therefore, the effective method to improve performance of RFEC SCC detection is designing a novel sensor and detection system, which is more sensitive to tiny axial crack.

\section{EXPERIMENT OF CONVENTIONAL RFEC NDT}

Fig. (2) shows conventional remote field eddy current sensor, which usually adopts a coaxial solenoid coil excited by sinusoid to generate exciting field, and adopts another solenoid coil placed at about 2 3 times pipes inner diameter to pick up disturbed electromagnetic field [5-7].

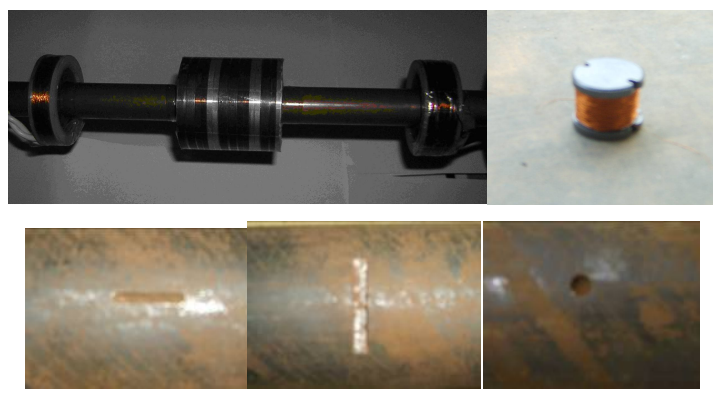

Fig. (2). Conventional sensor prototype of RFEC and defect.

To verify detection ability and sensitivity of conventional sensor, two classes defects are manufactured in a section of 
ferromagnetic tube with $70 \mathrm{~mm}$ inner diameter, $82 \mathrm{~mm}$ outer diameter and $6 \mathrm{~mm}$ wall thickness. First class defects consist of circumferential and axial crack with $10 \mathrm{~mm}$ length and 2 $\mathrm{mm}$ width, circular defect with $6 \mathrm{~mm}$ diameter; second class defect consist of circumferential and axial crack with $10 \mathrm{~mm}$ length and $0.5 \mathrm{~mm}$ width, circular defect with $3 \mathrm{~mm}$ diameter. All defects are manufactured with depth of $3 \mathrm{~mm}$, equal to $50 \%$ tube wall thickness.

Fig. (3) shows experiment results of first class defects. All of defects can be detected and identified clearly, although sensitivity to axial crack is relative lower than circumferential crack and circular defect. Fig. (4) shows experiment results of second class defects. With reduction of size of defect, detection ability to all of the defects decreases, especially for axial crack. Both amplitude and phase of detection coil for axial cracks reduce too low to identify axial crack.

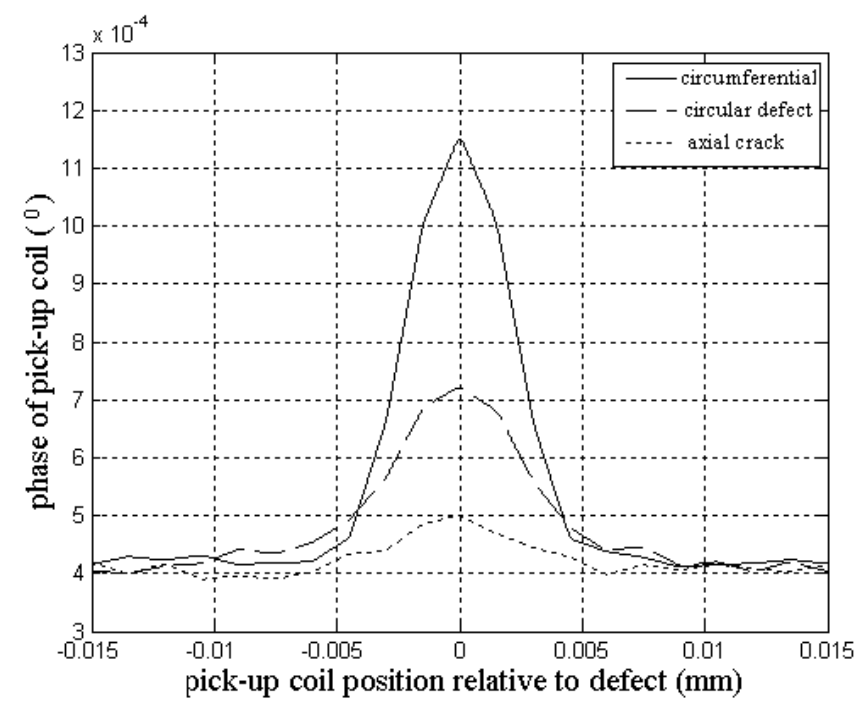

Fig. (3). Experiment results for first class defects.

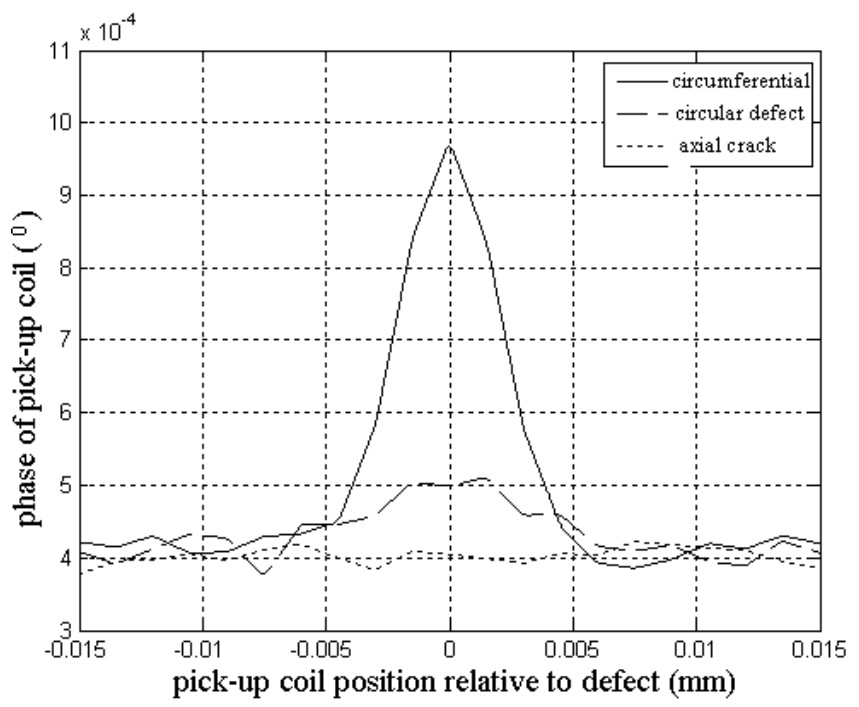

Fig. (4). Experiment results for second class defects.

Fortunately, to solve the detection ability for crack in different directions, rotating excitation method has been proposed for eddy current NDT and its applicability has been verified [8-10]. This type coil generates orthogonal magnetic field based on rectangular frame and two perpendicular windings on it. Unfortunately, it is not suitable for tubular structure, for its big air gap and un-uniform will cause serious problem for RFEC. The appropriate orthogonal pattern should be combined axial magnetic field and circumferential magnetic field for tubular structure.

\section{HIGH RESOLUTION RFEC DETECTION SYSTEM SIMULATION}

\subsection{Principle of Finite Element Method}

Maxwell equation is basis of electromagnetic simulation. For remote filed eddy current simulation, because its low frequency, displacement current will be omitted [11-13], therefore the Maxwell equation can be simplified as followed:

$\nabla \times \vec{H}=\vec{J}+\vec{J}_{S}$

$\nabla \times \vec{E}=-\frac{\partial \vec{B}}{\partial t}$

$\nabla \cdot \vec{B}=0$

where,

$\vec{J}_{s}$--- Source current density;

$\vec{J}$--- Eddy current density;

$\vec{B}$--- Magnetic flux density;

$\vec{H}$--- Magnetic field intensity;

$\vec{E}$--- Electric field intensity.

From Equation (1) $\sim(3)$, let $\vec{B}=\nabla \times \vec{A}$, where $\vec{A}$ is called magnetic potential vector, the final governing equation can be rewritten as:

$\nabla \times \frac{1}{\mu} \nabla \times \vec{A}=-\sigma \frac{\partial \vec{A}}{\partial t}+\vec{J}_{s}$

Deeply, for harmonic condition, and suggests $\nabla \cdot \vec{A}=0$, we can get :

$\left(\frac{1}{\mu}\right) \nabla^{2} \vec{A}=-\vec{J} S+j \omega \sigma \vec{A}$

Therefore, after mesh and discretion, magnetic potential vector $\vec{A}$ can be solved from equation (5). And then, magnetic flux density $\vec{B}$ and other quantity can be got.

\subsection{Simulation of High Resolution RFEC}

Finite element simulation parameters can be seen from Table 1, and Fig. (5) shows simulation model and mesh.

Fig. (6) shows simulation results of time-varying magnetic flux density in remote field zone. In remote field zone, the magnetic flux density is orthogonal also like the excitation, and can be divided to in-phase part and quarter part, which can be sensitive to defect in different directions.

Fig. (7) shows radial direction magnetic flux density around the axial crack. Significant change around crack confirms that the remote field zone can be use for tiny axial crack detection. 
Table 1. Parameters of finite element simulation.

\begin{tabular}{|c|c|c|}
\hline \multicolumn{3}{|c|}{ Tube } \\
\hline Outer diameter $[\mathrm{mm}]$ & & 82 \\
\hline Inner diameter [mm] & & 70 \\
\hline Wall thickness [mm] & & 6 \\
\hline Relative permeability & & 329.5 \\
\hline Conductivity [s/m] & & $0.5 \times 10^{7}$ \\
\hline OD Defect [mm] & \multicolumn{2}{|c|}{$\begin{array}{l}\text { Axial crack } 10 * 2 * 1.8 \text { (length, width, depth) } \\
\text { Located away from exciting coil } 246 \mathrm{~mm} \\
\text { ( } 3 \text { times pipe ID) }\end{array}$} \\
\hline \multicolumn{3}{|c|}{ Sensor } \\
\hline \multicolumn{2}{|c|}{ Exciting coil current density $\left[\mathrm{A} / \mathrm{m}^{2}\right]$} & $\begin{array}{c}\text { Axial }: 8.2 \times 10^{6} ; \\
\text { circumferential: } 1.6 \times 10^{6}\end{array}$ \\
\hline \multirow{2}{*}{ Exciting waveform } & Pattern & $30 \mathrm{~Hz}$ sinusoid \\
\hline & Phase & Axial: $0^{\circ} ;$ circumferential: $90^{\circ}$ \\
\hline
\end{tabular}

\section{HIGH RESOLUTION RFEC DETECTION SYSTEM DESIGN}

The proposed high resolution detection system is showed in Fig. (8). Signal generation unit uses AD7008 DDS chip to generate two orthogonal excitation waveforms. The power amplifier unit uses OPA548 to amplifier the orthogonal signal and applied to exciting coil. Fig. (9) shows the novel high resolution sensor.

\subsection{Excitation}

Conventional RFEC sensor always uses a solenoid coil to generate axial excitation. Therefore, only another coil which can get circumferential excitation is needed to generate orthogonal excitation. For the excitation configuration showed in Fig. (10), because of "skin effect" theory, when $\mathrm{AC}$ current was applied between the inside conductor's two

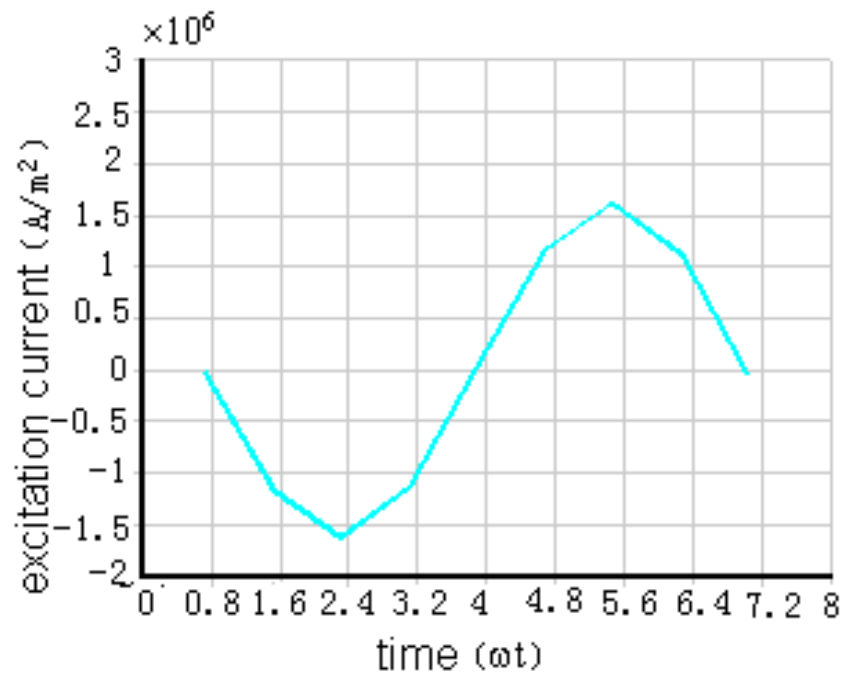

ends, the current will focus on conductor's out side [14-16], then can obtain spatial circumferential excitation. Important phenomenon "phase knot" is used for test and verification. From the curve shown in Fig. (10), the remote field zone begin from about 3 times of inner diameter, and means it is an appropriate for RFEC detection.

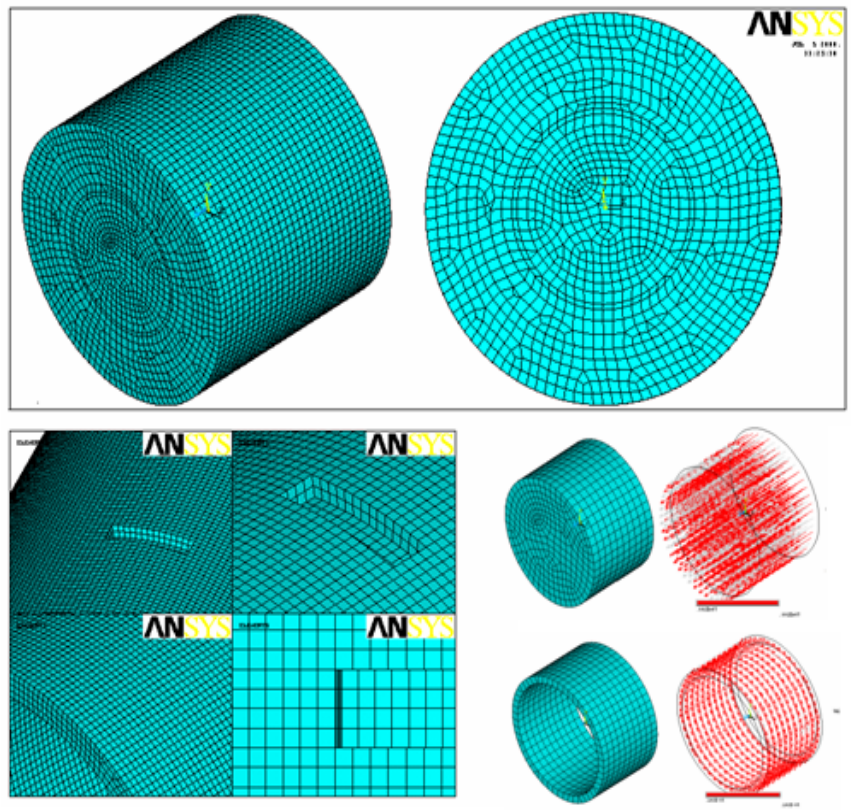

Fig. (5). Simulation model and orthogonal magnetic field.

\subsection{Detection Coil}

Differential detection coil is popular in RFEC tiny crack testing [17, 18]. Conventional differential mode coils has disadvantage such as susceptibility to jitter or off-center and difficulty to ensure windings symmetric. From the simulation results, to maximize the use of orthogonal excitation, the detection coil should be sensitive to radial perturbation field, and differential radial field detection coil shown in Fig. (11) is used to improve the detection ability and sensitivity $[2,19]$.

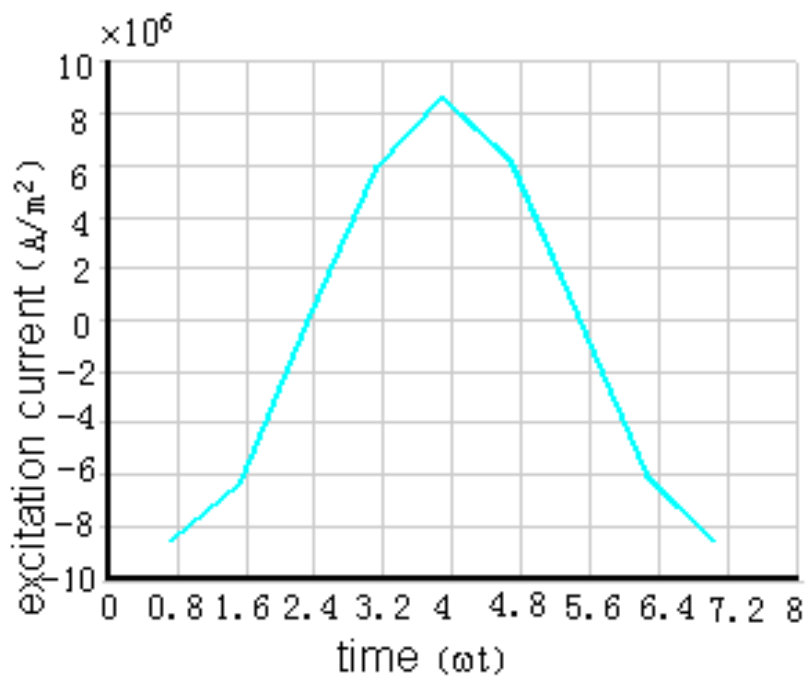

Fig. (6). Time-varying magnetic flux density. 


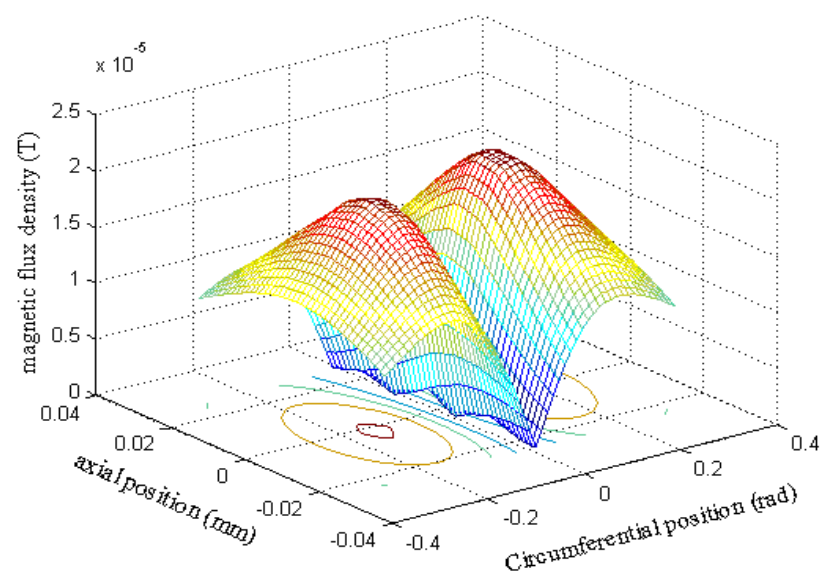

Fig. (7). Radial direction magnetic flux density.

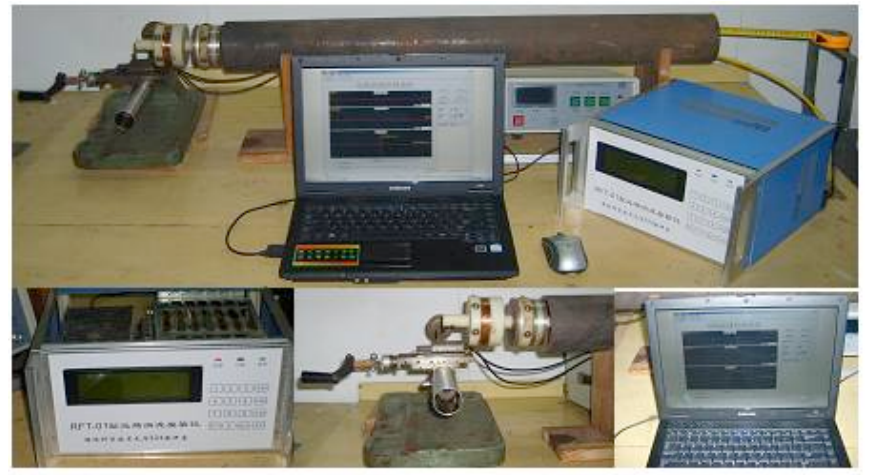

Fig. (8). Principle and photography of detection system.

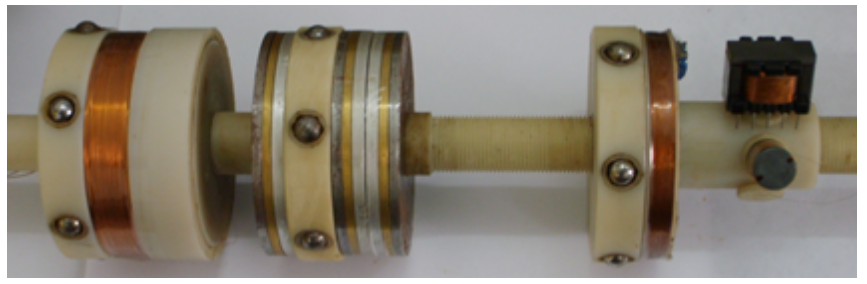

Fig. (9). Photography of high resolution sensor.
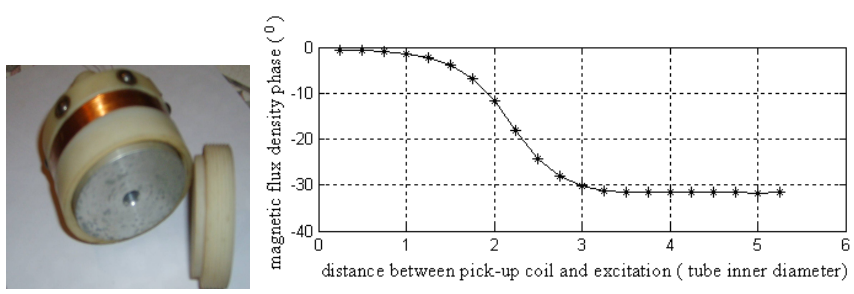

Fig. (10). Photography of exciting coil and "phase knot".
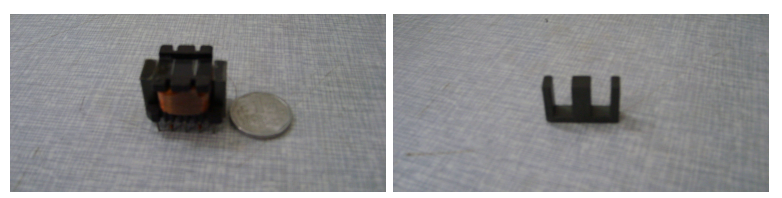

Fig. (11). Differential radial field detection coil.

The detection coil mainly relies on the symmetry of the magnetic core and its basic principle can be summarized as followed: Firstly, in the case of defect free, according to magnetic path theory and its symmetry, the magnetic flux will be leaded from one of the shaft to another shaft, and no flux will enter the winding; Secondly, when crack locates at one side of shafts, the coil's symmetry will be broken, then some magnetic flux will change its path to the central winding, as shown in Fig. (12); Lastly, when crack locates at another side of shafts, some magnetic flux will also change its path to the winding, except for its direction is inverse, as shown in Fig. (13).

\subsection{Experimental Condition}

(1) Frequency: Remote field eddy current always operate at low frequency, for example, $10 \mathrm{~Hz} 120 \mathrm{~Hz}$. Main criteria to select frequency is signal strength and place where remote field zone begin. From experimental test, $30 \mathrm{~Hz} \sim 60 \mathrm{~Hz}$ should be appropriate frequency, for under these frequency, a suitable detection signal can be obtained and the remote field zone is about $2 \sim 3$ times of pipe diameter. At last, 60 $\mathrm{Hz}$ is chosen to get a better signal to noise ratio.

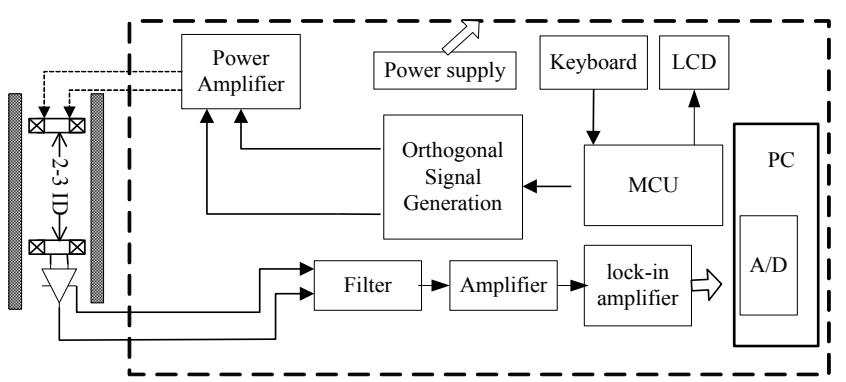

(2) Exciting power: Because of the "pass through twice" character of remote field eddy current, it is not an appropriate method to increase detection signal strength by increasing exciting power; exciting power should be set at an appropriate level. According to skin effect equation:

$\theta^{\prime}=2 h * \sqrt{\pi f \mu \sigma}=2 h * \sqrt{\pi f \sigma} * \sqrt{\mu}$

Threshold of exciting power can be determined by change of phase. Finally exciting power of axial excitation is chosen as $1.1 \mathrm{~A}$, and circumferential excitation 1.7 A.

(3) Sensor length reducing: In conventional RFEC, detection coil always fixed at $4 \sim 5$ times tube diameter to avoid the jitter or off-center of sensor, and forbidden the ability to pass through the bend and elbow. Using shielding is a popular method to solve the problem. By experimental test, we use the mode: steel first, copper second, aluminum last, which can be seen in Fig. (14). After using shielding, the remote field zone begins from 1.8 inner diameters. And 2.3 inner diameter should be appropriate, because of irregular of pipe and problem caused by inaccuracy of transmission gear.

\section{EXPERIMENT RESULTS}

Fig. (15) shows reference materials for experiment test. The specimen is a section of ferromagnetic tube with $70 \mathrm{~mm}$ inner diameter, $82 \mathrm{~mm}$ outer diameter and $6 \mathrm{~mm}$ wall thickness. Three type defects are used for test. First class defect consists of 

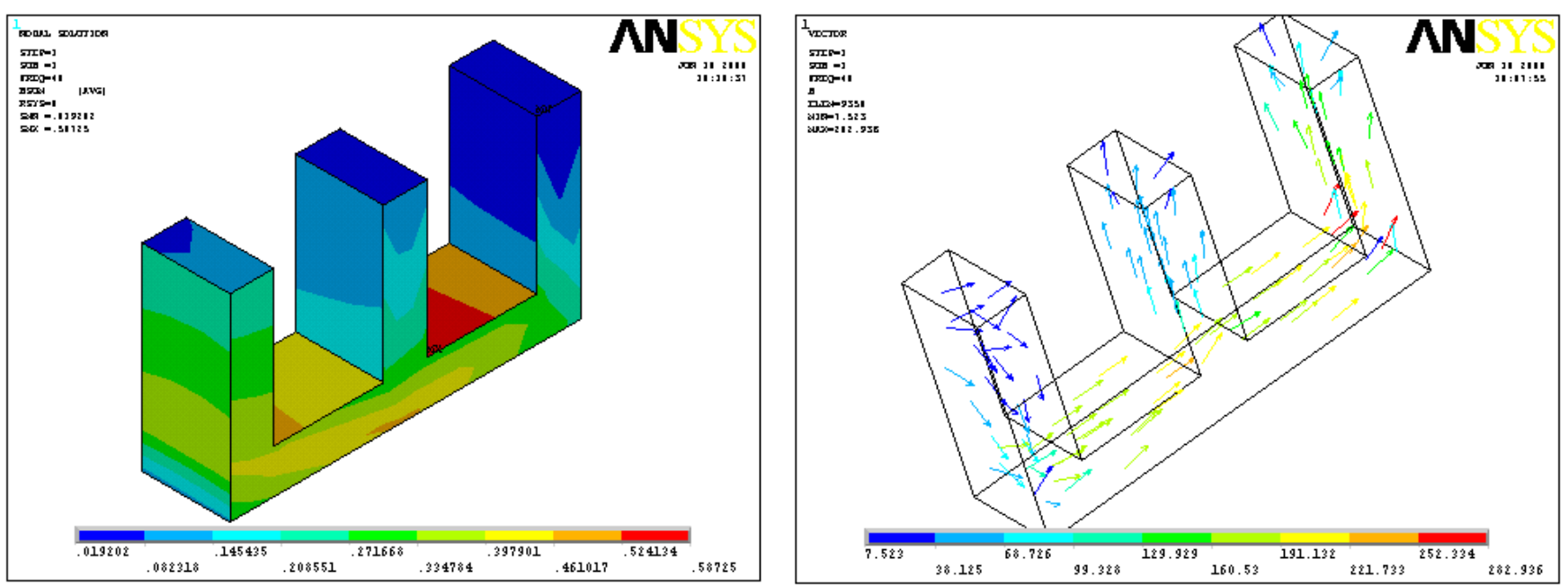

Fig. (12). Magnetic flux entering from bottom.
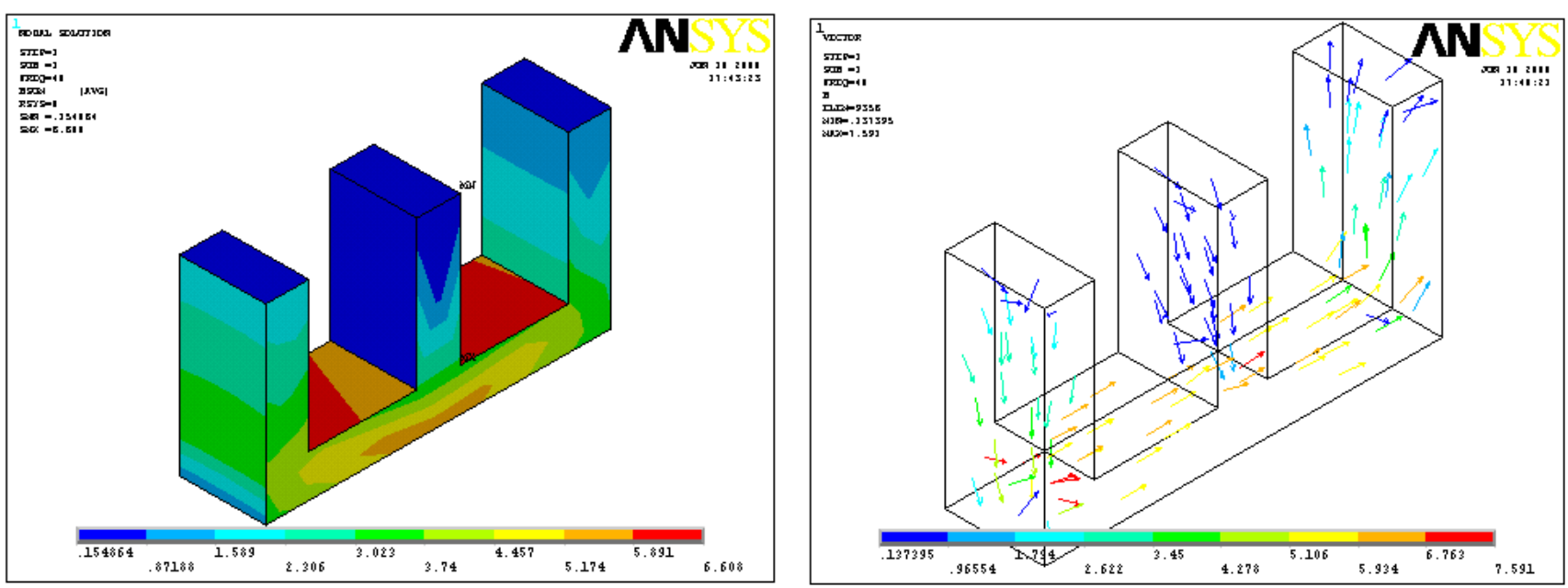

Fig. (13). Magnetic flux entering from top.

manufactured axial cracks with $10 \mathrm{~mm}$ length, $0.5 \mathrm{~mm}$ width, and different depth of $15 \%, 20 \%, 40 \%, 60 \%$ tube wall thickness; Second type is two parallel axial crack with $3 \mathrm{~mm}$ separation distance; Third type defect is a real stress corrosion crack.
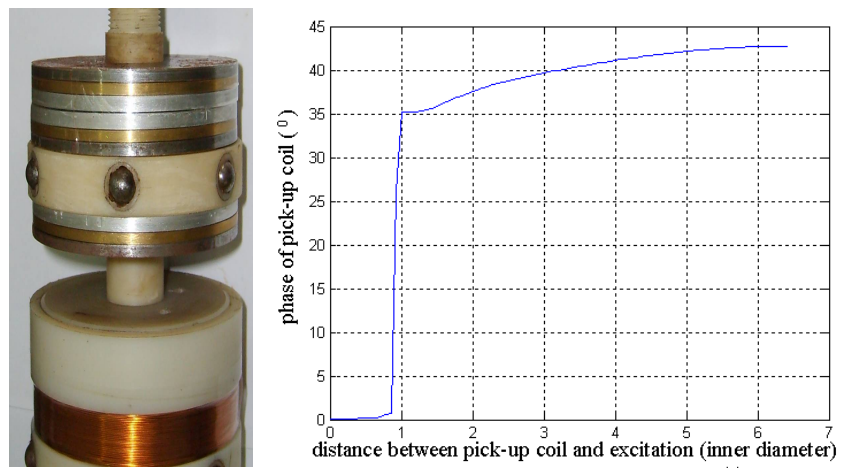

Fig. (14). Shielding pattern and "drag test" experiment result.

\subsection{Results of Axial Cracks with Different Depth}

Figs. $(16,17)$ show experiment results of axial cracks with different depth. From the amplitude and phase of detection coil, except for defects with depth of $15 \%$ wall thickness, all of other defects can be detected; especially, the proposed high resolution detection system can detect and identify axial crack with depth of $20 \%$ wall thickness. Compared with the section 2, the detection ability is improved significantly.
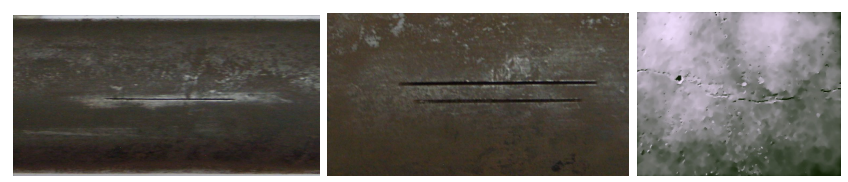

Fig. (15). Three type defects on test specimen.

\subsection{Results of Two Parallel Axial Cracks}

Figs. $(18,19)$ show experiment results of two parallel axial cracks. The amplitude and phase can detect the crack clearly, and from the contour plot of amplitude, the shape of crack can be evaluation, although the phase failed to discrimination of two cracks.

\subsection{Results of Real Stress Corrosion Crack}

Figs. $(\mathbf{2 0}, \mathbf{2 1})$ show experiment results of the real stress corrosion crack. From the 3D plot of detection coil, the proposed method can detect stress corrosion crack obviously, 
but it is not easily to determine the shape of the crack even in the contour plot. Fortunately, we can use the contour plot of phase to identify shape of the crack, for phase is usually chosen to determine defect in RFEC testing.

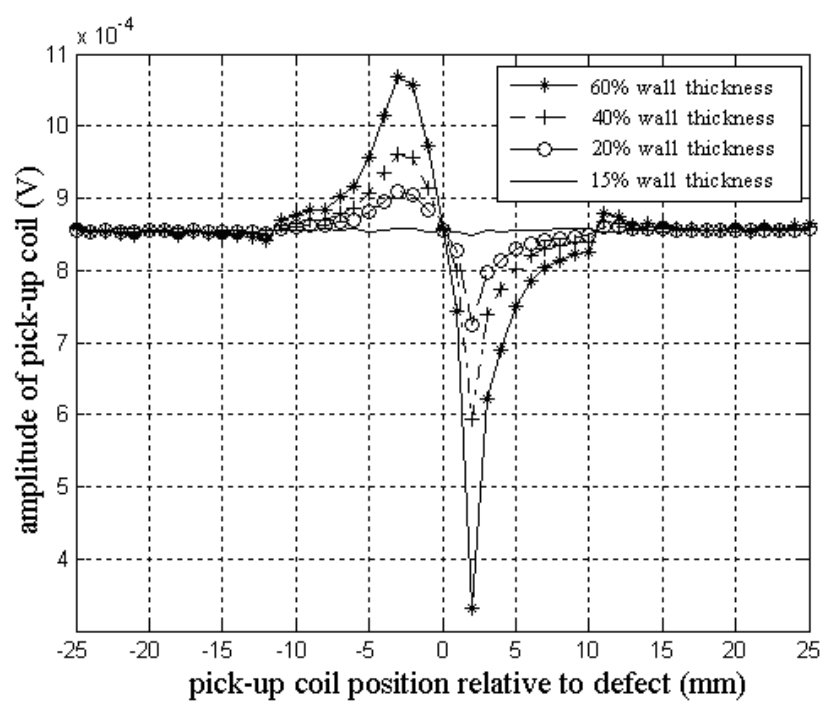

Fig. (16). Amplitude of detection coil for axial cracks.

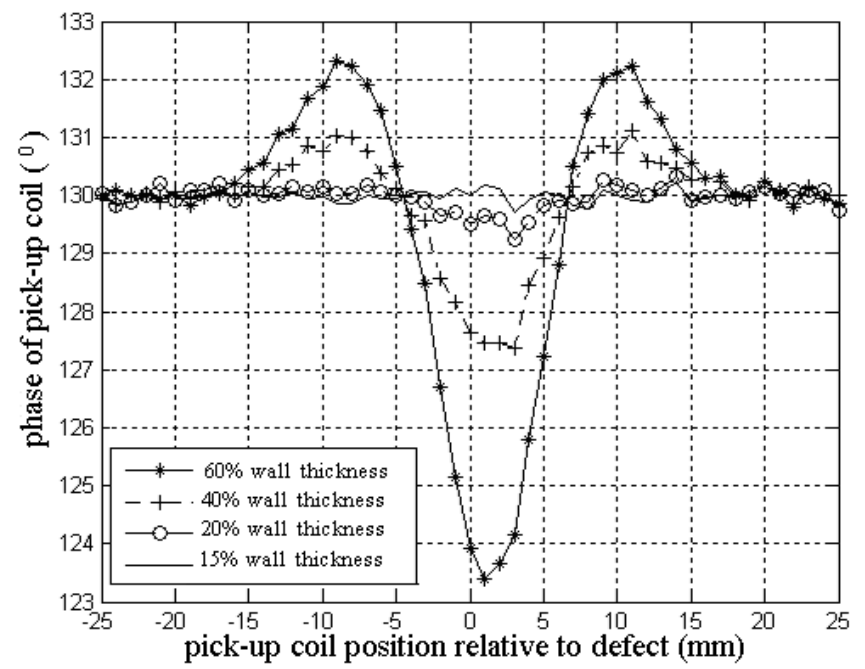

Fig. (17). Phase of detection coil for axial cracks.

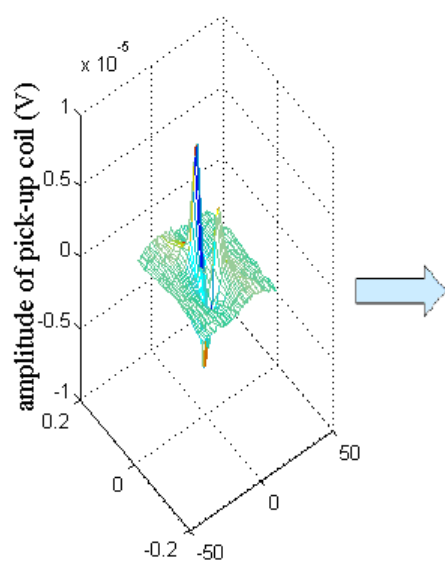

(a) $3 \mathrm{D}$ plot

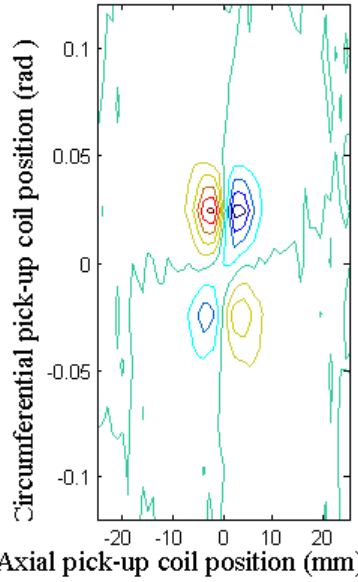

(b) contour plot

Fig. (18). Amplitude of detection coil for parallel cracks.

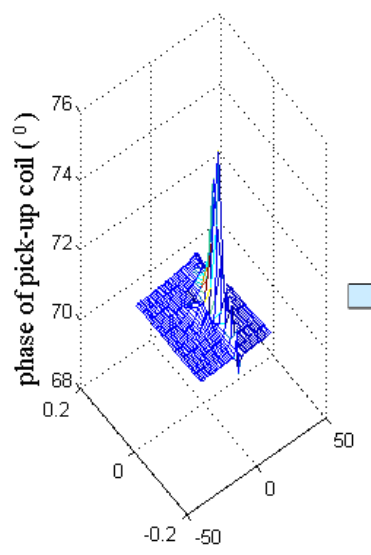

(a) 3D plot

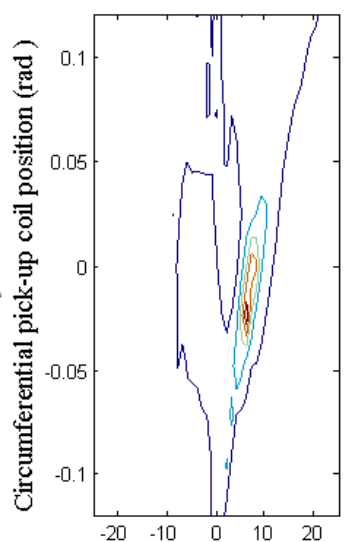

Axial pick-up coil position (mm)

(b) contour plot
Fig. (19). Phase of detection coil for parallel cracks.

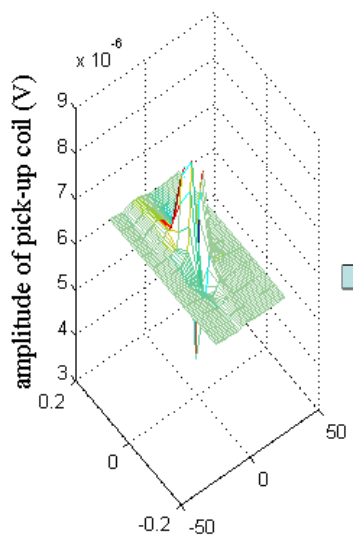

(a) $3 \mathrm{D}$ plot

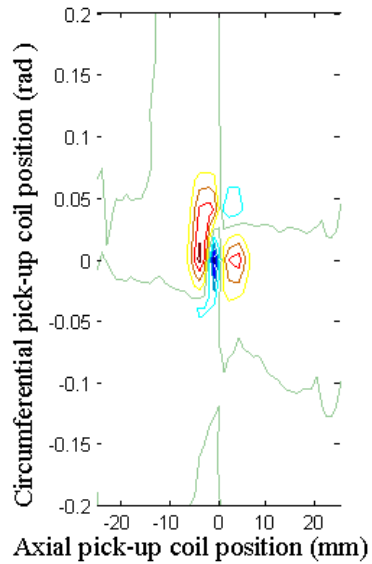

(b) contour plot
Fig. (20). Amplitude of detection coil for real SCC.

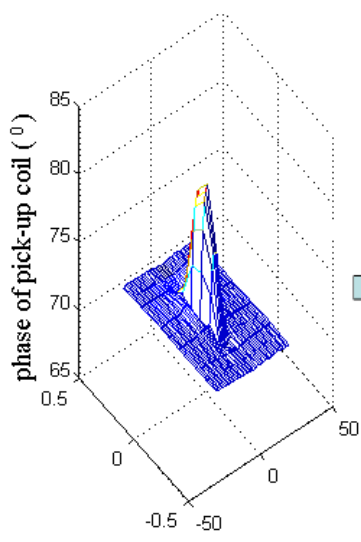

(a) $3 \mathrm{D}$ plot

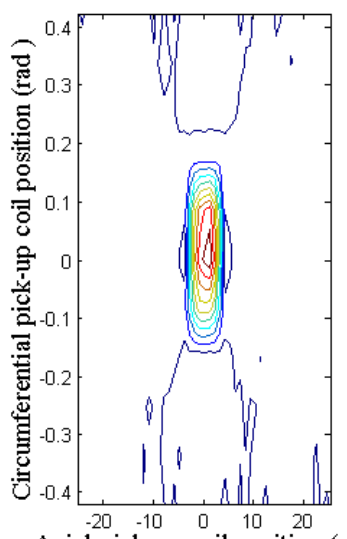

Axial pick-up coil position (mm)

(b) contour plot
Fig. (21). Phase of detection coil for real SCC.

\section{CONCLUSION}

Stress corrosion crack is not easily to detect using conventional remote field eddy current method. It is mainly because of that conventional RFEC sensor only using axial magnetic filed excitation, and detection coil usually is designed to detect axial disturbed magnetic field. A novel 
high resolution remote field eddy current detection system is proposed and verified. Firstly, the poor detection ability to axial crack of conventional RFEC detection system is discussed with detailed. Secondly, appropriate orthogonal excitation and detection coil mode is design and tested. Experiment results show that proposed novel detection system has high resolution to various type of axial cracks, especially to stress corrosion crack which is difficult to detect by conventional system, and the shape and size of stress corrosion crack can be evaluated by combination utilize the amplitude and phase of detection coil.

\section{ABOUT THE AUTHORS}

First Author Xu Xiaojie, Institute of Information and Navigation of Air Force Engineering University, Ph.D. The author's major is Eddy Current Non-Destructive Testing and Signal Processing. 5 papers received by EI or SCI and 1 patents had been published.

Second Author Jia Yueling, Institute of Equipment management and Engineering of Air Force Engineering University. The author's major is Equipment Engineering and Computer Simulation.

Third Author Zang Zhanbin, Institute of Information and Navigation of Air Force Engineering University. The author's major is Techtronic Engineering and Signal Processing.

\section{CONFLICT OF INTEREST}

The authors confirm that this article content has no conflict of interest.

\section{ACKNOWLEDGEMENTS}

This work was financially supported by The Chinese National Natural Science Fund (51107148) and The Natural Science Foundation of Shanxi Province (2011JQ7006).

\section{REFERENCES}

[1] N. Kobayashi, S. Ueno, S. Nagai, M. Ochiai, and N. Jimbo, "Remote field eddy current testing for steam generator inspection of fast reactor", Nuclear Engineering and Design, vol. 241, no. 12, pp. 4643-4648, 2011.

[2] X. Xu, M. Liu, Z. Zhang, and Y. Jia, "A novel high sensitivity sensor for remote field eddy current non-destructive testing based on orthogonal magnetic field", Sensors, vol. 14, no. 12, pp. 2409824115,2014

[3] X. Xu, and F. Luo. "Optimal sensor design and digital signal processing techniques for remote field eddy current testing", Insight, vol. 48, no. 6, pp. 421-425,2006.

[4] D.L. Atherton, "Remote field eddy current inspection", IEEE Transactions on Magnetic, vol. 31, no. 1, pp. 4142-4147, 1995.

[5] Y. Kim, and S. Lee, "Eddy current probes of inclined coils for increased detectability of circumferential cracks in tubing", $N D T \&$ E International, vol. 49, pp. 77-82, 2012.

[6] S. Smith, and D.L. Atherton, "Longitudinal AC interactions with axial slits in steel pipes", Research on Nondestructive Evaluation, vol. 7, pp. 203-214, 1996.

[7] D. Kim, L. Upda, and S. Upda, "Remote field eddy current testing for detection of stress corrosion cracks in gas transmission pipelines". Materials Letters, vol. 58, no. 15, pp. 2102-2104, 2004.

[8] J. Xin, N. Lei, L. Udpa, and S.S. Udpa, "Rotating field eddy current probe with bobbin pickup coil for steam generator tubes inspection", NDT \& E International, vol. 54, pp. 45-55, 2013.

[9] T.E. Capobinaco, "Rotating field eddy current probe for characterization of cracking in non-magnetic tubing", Technology Report, pp. 1-58, 1998.

[10] R. Grimberg, L. Upda, and S. Upda, "A novel rotating magnetic field eddy current transducer for the examination of fuel channels in PHWR nuclear power plants". Review of Progress in QNDE, vol. 24, pp. 471-478, 2005.

[11] Z. Chen, M. Rebican, K. Miya, and T. Takagi "Three dimensional simulation of remote field ECT using the Ar method and a new formula for signal calculation", Research in Nondestructive Evaluation, vol. 16, pp. 35-53, 2005.

[12] N. Yuma, S. Perrine, and K. Mizuno, "Numerical modeling of general cracks from the viewpoint of eddy current simulations", NDT \& E International, vol. 40, pp. 577-583, 2007.

[13] H.S. Lopez, M. Poole, and S. Crozier, "Eddy current simulation in thick cylinders of finite length induced by coils of arbitrary geometry", Journal of Magnetic Resonance, vol. 207, no. 2, pp. 251-261, 2010.

[14] P. Wang, Z. Fu, and T. Ding, "A frameless eddy current sensor for cryogenic displacement measurement", Sensors and Actuators A: Physical, vol. 159, no. 1, pp. 7-11, 2010.

[15] P. Rolicz, "Eddy currents generated in a system of two cylindrical conductors by a transverse alternating magnetic field", Electric Power Systems Research, vol. 79, no. 4 pp. 295-300, 2009.

[16] X. Mao, and Y. Lei, "Analysis of eddy current interaction between a parallel coil and a ferromagnetic pipe with remanence", $N D T \& E$ International, vol. 60, pp. 121-126, 2013.

[17] L.S. Rosado, T.G. Santos, and P.M. Ramos, P. Vilaça, M. Piedade, "A differential planar eddy currents probe: fundamentals, modeling and experimental evaluation", NDT \& E International, vol. 51, pp. 85-93, 2012.

[18] R.R. Robaina, H.T. Alvarado, and J.A. Plaza, "Planar coil-based differential electromagnetic sensor with null-offset", Sensors and Actuators A: Physical, vol. 164, no. 1, pp. 15-21, 2010.

[19] Y. Sun, D. Roach, and H. Zhu, "New advances in detecting cracks in raised-head fastener holes using rotational remote field eddy current technique", ASNT Fall Conference, 2005, pp. 17-21. 